\title{
Numerical Modeling of Pool Fires Using LES and Finite Volume Method for Radiation
}

\author{
SIMO HOSTIKKA
}

VTT Building and Transport, Espoo, Finland

simo.hostikka@vtt.fi

\author{
KEVIN B. McGRATTAN and ANTHONY HAMINS \\ Building and Fire Research Laboratory \\ National Institute of Standards and Technology \\ Gaithersburg, Maryland, USA
}

\begin{abstract}
The thermal environment in small and moderate-scale pool flames is studied by Large Eddy Simulation and the Finite Volume Method for radiative transport. The spectral dependence of the local absorption coefficient is represented using a simple wide band model. The predicted radiative heat fluxes from methane/natural gas flames as well as methanol pool burning rates and flame temperatures are compared with measurements. The model can qualitatively predict the pool size dependence of the burning rate, but the accuracy of the radiation predictions is strongly affected by even small errors in prediction of the gas phase temperature.
\end{abstract}

KEYWORDS: Large Eddy Simulation, radiation modeling, pool flames, radiative fraction, burning rate

\section{INTRODUCTION}

Use of computational fluid dynamics (CFD) for fire modeling has increased dramatically during the last few years, mainly due to the increased computational power and the development of fire specific computer codes. Most models have used Reynolds-averaged Navier-Stokes solvers combined with turbulence models and relatively simple combustion models. On the other hand, highly complex combustion models with detailed solution of gas phase flows have been presented by the combustion research community for many years. The exchange of information and experiences between the fire and combustion communities has been rare, mainly due to the very different physical length scales over which the models are applied.

The objective of the paper is to explore the application of a Large Eddy Simulation (LES) technique to pool flames, which are typical source terms in the fire safety engineering. LES technique is used to model pool flames characterized by a physical scale that lies between combustion research and compartment fire research. Combustion is modeled using a mixture fraction approach, which assumes that the reaction takes place in a flame sheet that moves with the three dimensional turbulent flow. The focus of the paper is in the modeling of the transport of thermal radiation using a Finite Volume Method combined with a simple wide band model for the spectral dependence of the local 
absorption coefficient. The model is used to predict the radiation from methane/natural gas flames for burner diameters between $10 \mathrm{~cm}$ and $100 \mathrm{~cm}$. Another application tested by the models is the prediction of the burning rate of circular methanol pools, ranging from $1 \mathrm{~cm}$ to $100 \mathrm{~cm}$ diameter. The time averaged flame temperatures are compared with measurements in the case of the $30 \mathrm{~cm}$ methanol pool. Measurements of distribution of radiative heat flux, the fuel mass burning rate, and the gas-phase temperature are described here in detail.

\section{MODEL DESCRIPTION}

\section{Hydrodynamic model}

The fluid flow is modeled by solving the conservation equations for mass, mixture fraction, momentum and energy in a low Mach number form. The details of the model have been described previously $[1,2]$ and will not be repeated here. A full description can be found in Ref. [3]. The effect of the flow field turbulence is modeled using LES, in which the large scale eddies are computed directly and the sub-grid scale dissipative processes are modeled. An explicit, second-order accurate scheme is used for the time integration.

\section{Combustion model}

The combustion model is based on the assumption that the combustion is mixingcontrolled. This implies that all species of interest can be described in terms of the mixture fraction $Z$, a conserved scalar variable. Heat from the reaction of fuel and oxygen is released along an infinitely thin sheet where $Z$ takes on its stoichiometric value as determined by the solution of the transport equation for $Z$. The heat release rate per unit area of flame surface is

$$
\dot{q}_{C}^{\prime \prime}=\left.\Delta H_{O} \frac{d Y_{O}}{d Z}\right|_{z<z_{f}}(\rho D) \nabla Z \cdot \mathbf{n}
$$

where $\Delta H_{O}$ is the energy released per unit mass of oxygen consumed, being almost constant for a wide range of fuels [4], and $\mathbf{n}$ is the unit normal facing outward from the fuel [5]. Note that both $d Y_{O} / d Z$ and $\nabla Z \cdot \mathbf{n}$ are negative. Expressing the heat release rate in terms of the oxygen consumption is very useful because the chemical composition of the fuel is not well characterized in compartment fires.

\section{Thermal radiation model}

The Radiative Transport Equation (RTE) for a non-scattering gas is

$$
\mathbf{s} \cdot \nabla I_{\lambda}(\mathbf{x}, \mathbf{s})=\kappa_{\lambda}(\mathbf{x})\left[I_{b}(\mathbf{x})-I_{\lambda}(\mathbf{x}, \mathbf{s})\right]
$$

where $I_{\lambda}(\mathbf{x}, \mathbf{s})$ is the radiation intensity at wavelength $\lambda, I_{b}(\mathbf{x})$ is the source term given by the Planck function, $\mathbf{s}$ is the unit normal direction vector and $\kappa_{\lambda}(\mathrm{x})$ is the spectral absorption coefficient. In practical simulations, the spectral dependence cannot be solved accurately. Instead, a simple wide band model is developed by dividing the radiation spectrum into a relatively small number of bands. The band specific RTE's are 


$$
\mathbf{s} \cdot \nabla I_{n}(\mathbf{x}, \mathbf{s})=\kappa_{n}(\mathbf{x})\left[I_{b, n}(\mathbf{x})-I_{n}(\mathbf{x}, \mathbf{s})\right], \quad n=1 \ldots N
$$

where $I_{n}$ is the intensity integrated over the band $n$ and $\kappa_{n}$ is the appropriate mean absorption coefficient. The source term can be written as a fraction of the blackbody radiation

$$
I_{b, n}(\mathbf{x})=F_{n}\left(\lambda_{\min }, \lambda_{\max }\right) \sigma T(\mathbf{x})^{4}
$$

where $\lambda_{\min }$ and $\lambda_{\max }$ are the limits of the band and $\sigma$ is the Stefan-Boltzmann constant. The calculation of factors $F_{n}$ is explained, for example, in Ref. [6]. A narrow-band model RADCAL [7] is combined with the radiation solver for the calculation of the band mean absorption coefficients $\kappa_{n}$. When the intensities corresponding to the bands are known, the total intensity is calculated by summation $I(\mathbf{x}, \mathbf{s})=\Sigma I_{n}$.

Even with a reasonably small number of bands, the solution of the RTE's is very time consuming. Fortunately, in most large-scale fire scenarios soot is the most important combustion product affecting thermal radiation. As the radiation spectrum of soot is continuous, it is possible to assume that the gas behaves as a gray medium. The spectral dependence is then lumped into one effective absorption coefficient $(N=1)$ and the source term is given by the blackbody radiation intensity. For the computation of the effective absorption coefficients with RADCAL, a path length must be defined. Here, it is calculated as $0.1 \times 3.6 \times L_{x} L_{x} L_{x} /\left(L_{x} L_{y}+L_{x} L_{z}+L_{y} L_{z}\right)$ which is one tenth of the mean beam length of the computational domain, with dimensions $L_{x}, L_{y}$ and $L_{z}$. In case of the wide band model, the Planck mean absorption coefficients are used as band mean coefficients. The limits of the bands are selected to give an accurate representation of the most important radiation bands of $\mathrm{CO}_{2}$ and water. The absorption coefficients are tabulated as a function of mixture fraction and temperature. During the simulation, the local absorption coefficient is found by a look-up table.

To retain computational simplicity and applicability to the different length scales, the soot formation and oxidation processes are not modeled. Instead, the soot concentration is obtained by assuming a certain conversion factor (yield) of soot. The local soot concentration is based on the value of mixture fraction alone. This is not a very good approximation in general, as the soot chemistry depends on the scale of the problem. However, it is consistent with the relatively simple combustion model adopted. A state relation between soot and mixture fraction is created, and the effect of soot is taken into account during the tabulation of absorption coefficients.

Numerical experiments found that six bands are usually enough $(N=6)$ for fire applications. If the absorption of the fuel is known to be important, separate bands should be reserved for the fuel, and the total number of bands is ten $(N=10)$. For simplicity, the fuel is assumed to be $\mathrm{CH}_{4}$. The limits of the bands are shown in Table 1 below. High temperature spectral information is not currently available for methanol, but the difference between its values and those of methane are expected to be relatively insignificant for the purpose of this investigation.

Equation (3) is solved using techniques similar to those for convective transport in finite volume methods for fluid flow [8], thus its name is the Finite Volume Method (FVM). To 
obtain the discretized form of the RTE, the unit sphere is divided into a finite number of solid angles. The distribution of angles is based on empirical rules that try to produce equal solid angles $\delta \Omega^{l}=4 \pi / N_{\Omega}$, where $N_{\Omega}$ is the total number of control angles. In each grid cell a discretized equation is derived by integrating Eq. (3) over the cell volume $V_{i j k}$ and the control angle $\delta \Omega_{l}$, to obtain

$$
\int_{V_{i j k}} \int_{\partial \Omega^{l}} \mathbf{s} \cdot \nabla I_{n}^{l} d \Omega d V=\int_{V_{i j k}} \int_{\partial \Omega^{l}} \kappa_{n}(\mathbf{x})\left[I_{b, n}(\mathbf{x})-I_{n}^{l}(\mathbf{x}, \mathbf{s})\right] d \Omega d V
$$

The volume integral on the left hand side is replaced by a surface integral over the cell faces using the divergence theorem. Assuming that the radiation intensity is constant on each of the cell faces, the surface integral can be approximated by a sum over the cell faces. The cell face intensity is calculated from the neighboring cells using the so called "step scheme" which is equivalent to a first order upwind scheme. The numerical diffusion caused by the low order scheme is actually used to smooth out the intensity field. Therefore, FVM suffers from the ray effects to a smaller extent than the more commonly used ray tracing approaches. The solution method of the resulting system is based on an explicit marching sequence [9], in which the marching direction depends on the propagation direction of the radiation intensity, and no iterations are needed.

Table 1. - Limits of the spectral bands. $v$ and $\lambda$ are the wave number and wavelength.

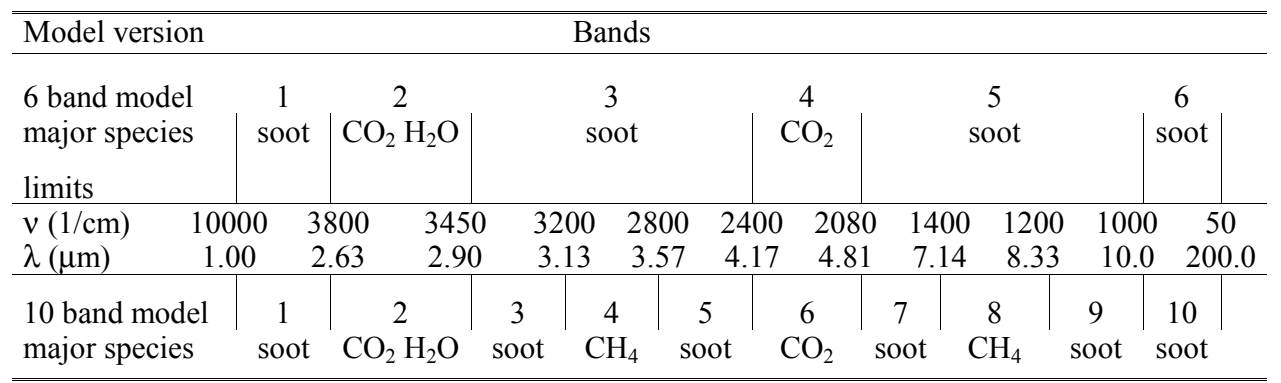

\section{Pyrolysis model for liquid fuels}

The rate at which liquid fuel evaporates when burning is a function of the liquid temperature and the concentration of fuel vapor above the pool surface. Equilibrium is reached when the partial pressure of the fuel vapor above the surface obtains equilibrium as determined by the Clausius-Clapeyron expression

$$
p_{c c}=p_{0} \exp \left[\frac{\Delta H_{v} M_{f}}{R}\left(\frac{1}{T_{s}}-\frac{1}{T_{b}}\right)\right]
$$

where $\Delta H_{v}$ is the heat of vaporization, $M_{f}$ is the molecular weight, $R$ is the universal gas constant, $T_{s}$ is the surface temperature, and $T_{b}$ is the boiling temperature of the fuel. For simplicity, the liquid fuel itself is treated like a thermally-thick solid for computing heat conduction into the pool. There is no consideration of convection within the liquid pool. 


\section{EXPERIMENTAL METHOD}

Methane, natural gas, and methanol fires were established in a quiescent environment using six different circular burners. A water-cooled, porous sintered-bronze $0.38 \mathrm{~m}$ burner and $1.0 \mathrm{~m}$ and $0.10 \mathrm{~m}$ water-cooled sand burners were used for the gaseous fuels. The liquid fuel burners were $0.10 \mathrm{~m}, 0.30 \mathrm{~m}$, and $1.0 \mathrm{~m}$ diameter. The sand burners had a $3 \mathrm{~mm}$ (outer diameter) copper water-cooling tube positioned $1 \mathrm{~cm}$ below the sand in the form of a loosely wound spiral. In addition, a copper water cooling tube was wrapped around the outside of the burner. The rate of gaseous fuel delivery was controlled using calibrated rotometers. The mass flow of the gaseous fuels was determined using a dry test meter and a stopwatch to measure the volume per unit time of fuel delivered to the burner and a thermocouple measurement of the ambient temperature. For the liquid fuel fires, the fuel was stored in a reservoir and gravity-fed to the burner at a controlled rate such that the fuel was maintained $1 / 2 \mathrm{~cm}$ below the rim of the burner. The mass delivery of liquid fuel was measured using a calibrated load-cell positioned under the fuel reservoir. Steady state burning conditions were established before measurements were initiated. A warm-up period of approximately $10 \mathrm{~min}$ was required for the experiments involving methanol and approximately $3 \mathrm{~min}$ for the gaseous fuels. The combined expanded measurement uncertainty for the burning rate was estimated as $4 \%$ for the gaseous fuels and $10 \%$ for methanol (as determined from the load cell measurements). All uncertainties are reported with a coverage factor of two, which is equal to two times the value of the standard deviation [10]. The experimental apparatus and method are described in more detail in Refs. [11] and [12], respectively.

Calibrated radiometers were used to measure the radiative flux along a cylindrical control surface surrounding the fire. Approximately 12 water-cooled $(2.5 \mathrm{~cm}$ diameter) wideangle $\left(150^{\circ}\right.$ view) radiometers were positioned in two rows. The first row was positioned on a vertical axis several burner diameters away from the fire. These radiometers were all oriented radially facing the fire. The second row was oriented facing up and positioned along a radius on the plane aligned with the burner surface. A schematic diagram of the set-up is shown in Fig. 1 and the experimental approach is reported in Ref. [12]. The gauges were calibrated using a secondary standard in the BFRL/NIST Radiometer Calibration Facility [13]. The measured signal was averaged for a $90 \mathrm{~s}$ period. The radiated power emitted by the fire was determined by integrating the measured spatial distributions of radiant flux. The radiative flux typically drops off very quickly in the radial direction, whereas in the vertical direction, the flux peaks at a vertical location equal to approximately $50 \%$ of the characteristic flame height and then drops to small values above the visible flame tip. The uncertainty (with a coverage factor of two) in the radiative flux measurement is estimated as $10 \%$. The radiative heat loss fraction $\left(\chi_{R}\right)$ was determined by dividing the measured radiant power by the idealized measured fire heat release rate. The uncertainty in the value of $\chi_{R}$ (with a coverage factor of two) is estimated as $11 \%$ for the gaseous fuels and $14 \%$ for methanol. Temperature measurements were conducted using fine $(75 \mu \mathrm{m})$ platinum/rhodium thermocouples and the results were corrected for radiative losses. The absolute magnitude of the uncertainty in the temperature is a function of the flame position; it typically was $5 \%$ to $10 \%$ (with a coverage factor of two). 


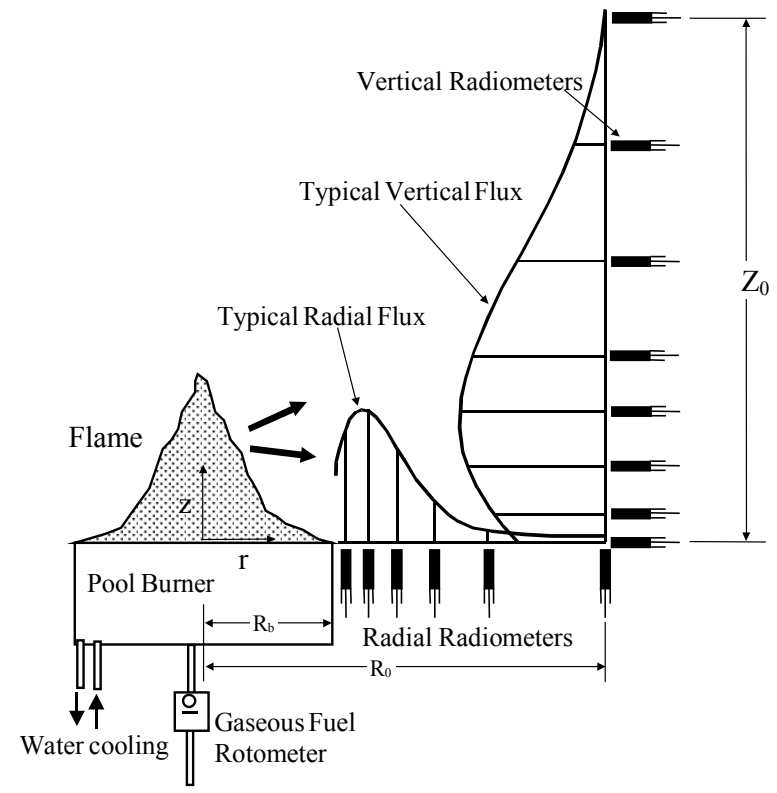

Fig. 1. - A schematic diagram of the set-up used to measure the radiative fraction.

\section{RESULTS AND DISCUSSION}

The model was used to predict the radiative heat flux from the methane / natural gas pool flames above the circular burners. The selected cases are summarized in Table 2, where $D$ is the burner diameter, $R_{0}$ is the radial position of the vertical row of radiometers, $\dot{m}_{F}^{\prime \prime}$ is the mass burning rate per burner area and $\dot{Q}^{\prime \prime}$ is the rate of heat release per burner area. The size of the simulation domain and the size of the computational grid cell just above the burner surface, $\delta x$, are also shown. The last two columns show the dimensionless heat release rates $Q_{D}{ }^{*}=\dot{Q} /\left(\rho_{\infty} T_{\infty} c_{p} D^{2} \sqrt{g D}\right)$ and $Q_{\delta x}{ }^{*}=(\dot{Q} / \mathrm{A}) /\left(\rho_{\infty} T_{\infty} c_{p} D^{2} \sqrt{g} \delta x\right)$ where $\dot{Q}$ is the heat release rate, $A$ is the burner area and $\rho_{\infty}, T_{\infty}$ and $c_{p}$ are the properties of the ambient air. $Q_{D} *$ is the standard dimensionless number, which characterizes the strength of the fire and $Q_{\delta x}{ }^{*}$ represents the resolution of the current grid, in proportion to the burning rate. A very low value of $Q_{\delta x}{ }^{*}$ indicates that the position of the flame near the burner surface cannot be resolved.

Table 2. - Summary of the simulated methane burner experiments.

\begin{tabular}{|c|c|c|c|c|c|c|c|c|}
\hline \multirow[b]{2}{*}{ Case } & \multicolumn{4}{|c|}{ Test configuration } & \multirow{2}{*}{$\begin{array}{c}\text { Simulated domain } \\
\mathrm{x} \times \mathrm{y} \times \mathrm{z} \\
\left(\mathrm{m}^{3}\right)\end{array}$} & \multicolumn{3}{|c|}{ Simulation parameters } \\
\hline & $\begin{array}{c}D \\
(\mathrm{~m})\end{array}$ & $\begin{array}{l}R_{0} \\
(\mathrm{~m})\end{array}$ & $\begin{array}{c}\dot{m}_{F}^{\prime \prime} \\
\left(\mathrm{g} / \mathrm{m}^{2} / \mathrm{s}\right)\end{array}$ & $\underset{\left(\mathrm{kW} / \mathrm{m}^{2}\right)}{\dot{Q}^{\prime \prime}}$ & & $\begin{array}{c}\delta x \\
(\mathrm{~cm})\end{array}$ & $Q_{D}^{*}$ & $Q_{\delta x}{ }^{*}$ \\
\hline A & 0.10 & 0.82 & 1.08 & 53.8 & $0.315 \times 0.21 \times 0.45$ & 0.525 & 0.12 & 0.67 \\
\hline B & 0.10 & 0.82 & 4.80 & 240 & $0.315 \times 0.21 \times 0.45$ & 0.525 & 0.53 & 3.0 \\
\hline $\mathrm{C}$ & 0.38 & 0.732 & 5.90 & 295 & $1.26 \times 0.84 \times 1.80$ & 2.1 & 0.34 & 1.8 \\
\hline $\mathrm{D}$ & 0.38 & 0.732 & 31.0 & 1550 & $1.26 \times 0.84 \times 2.20$ & 2.1 & 1.8 & 9.6 \\
\hline E & 1.0 & 1.00 & 1.25 & 62.4 & $2.50 \times 2.00 \times 3.50$ & 5.0 & 0.044 & 0.31 \\
\hline $\mathrm{F}$ & 1.0 & 0.80 & 4.12 & 206 & $2.50 \times 2.00 \times 4.50$ & 5.0 & 0.14 & 1.05 \\
\hline
\end{tabular}


For simplicity, both methane and natural gas flames were modeled as methane flames. The gas burners were modeled as a fuel inflow boundary, with surface temperature corresponding to the measured values. The vertical and top boundaries of the domain were open. The heat flux gauges were modeled as solid obstacles with a constant surface temperature of $+20{ }^{\circ} \mathrm{C}$. The size of the domain was selected such that the distance between the vertical boundaries and burner edge was at least one burner radius in the horizontal direction and eight burner radii in the vertical direction. The results were found insensitive to the distance of the vertical boundaries. The length of the domain was larger in $x$-direction than in $y$-direction, to allow the definition of the heat flux gauges. Cartesian, non-uniform grid was stretched in the vertical direction to have the smallest cell size just above the burner. The simulation of the steady state burning was run until the time averages for the predicted heat fluxes converged. The grid dependence of the predicted heat fluxes was studied by varying the grid size. Decreasing the cell size about $20 \%$ changed the results less than $20 \%$ and further reduction of the cell size to $50 \%$ of the original caused only minor changes. A large number (304) of control angles were used for the radiation solver to ensure accuracy of the solution. The results were found insensitive to the temporal accuracy of the radiation solver.

A soot conversion factor of $1.0 \%$ was assumed for all calculations. The resulting volume fractions inside the flame ranged from 0.05 to $0.12 \mathrm{ppm}$. The sensitivity of the predicted heat fluxes to the assumed conversion factor was studied for case D. Changing the conversion factor to $2.0 \%$ increased the heat fluxes $10 \%$ to $15 \%$, and correspondingly, setting it to zero decreased them by $15 \%$ to $20 \%$.

For each case described in Table 2, the radiative fraction of the heat release was calculated based on the predicted radiometer readings, using the same method, as in the interpretation of the experimental results. The measured and predicted radiative fractions are compared in Table 3. The predictions are systematically higher than the measurements. The accuracy of the spectral dependence ( 6 band, 10 band or gray gas) does not have a strong effect on the results. The most probable reason for the high radiative fractions is the overestimation of the flame temperatures. Predicted peak temperatures in the fluctuating flames were over $2100{ }^{\circ} \mathrm{C}$, which is close to the adiabatic flame temperature. The observed peak mean temperatures were $1300{ }^{\circ} \mathrm{C}(\mathrm{A}), 1700{ }^{\circ} \mathrm{C}$ (B), $1600^{\circ} \mathrm{C}(\mathrm{C}), 1100{ }^{\circ} \mathrm{C}$ (D), $800{ }^{\circ} \mathrm{C}$ (D) and $1400{ }^{\circ} \mathrm{C}(\mathrm{E})$. As can be seen, the temperatures are not systematically low or high, but in all cases the highest temperatures were found in the first computational cell above the burner.

Table 3. - Comparison of the measured and predicted radiative fractions for the methane/natural gas pool flames.

\begin{tabular}{cccccccc}
\hline \hline Case & \multirow{2}{*}{$\begin{array}{c}D \\
\dot{Q}^{\prime \prime}\end{array}$} & \multirow{2}{*}{$\chi_{R}$ Experiment } & \multicolumn{4}{c}{$\chi_{R}$ Model } \\
\cline { 5 - 8 } & $\left(\mathrm{kW} / \mathrm{m}^{2}\right)$ & & 6 band & 6 band, fine grid & 10 band & gray gas \\
\hline A & 0.10 & 53.8 & 0.10 & 0.11 & 0.13 & 0.13 & 0.12 \\
B & 0.10 & 240 & 0.16 & 0.22 & 0.21 & 0.20 & 0.22 \\
C & 0.38 & 295 & 0.15 & 0.19 & 0.24 & 0.19 & 0.20 \\
D & 0.38 & 1550 & 0.19 & 0.21 & 0.23 & 0.21 & 0.24 \\
E & 1.0 & 62.4 & 0.08 & 0.21 & 0.21 & 0.21 & 0.20 \\
F & 1.0 & 206 & 0.16 & 0.19 & 0.20 & 0.18 & 0.20 \\
\hline
\end{tabular}


The measured and predicted radial distributions of radiative flux are compared in Fig. 2 . The agreement is very good in general, but the heat flux is highly over estimated in two of the cases: the higher burning rate of the $10 \mathrm{~cm}$ burner (case B) and the lower burning rate of the $100 \mathrm{~cm}$ burner (case E). Similar trends can be found in Fig. 3, showing the vertical profiles. As the main application of the model is the simulation of compartment fires, it is encouraging to see that the high radiative fluxes have been predicted better than low fluxes. A few remarks should be made, when interpreting the results: First, the $100 \%$ errors in radiative heat flux may be caused by as low as $20 \%$ error in absolute temperature. Second, the lowest radiative fluxes here are of the same order of magnitude as the background radiation, and therefore very sensitive to the small errors in the boundary conditions and gas temperatures. Also, some errors are possible in the modeling of the heat flux gauges. For example, the view angle of the simulated gauges is always $180^{\circ}$, while the experimental gauges had a view angle of $150^{\circ}$.
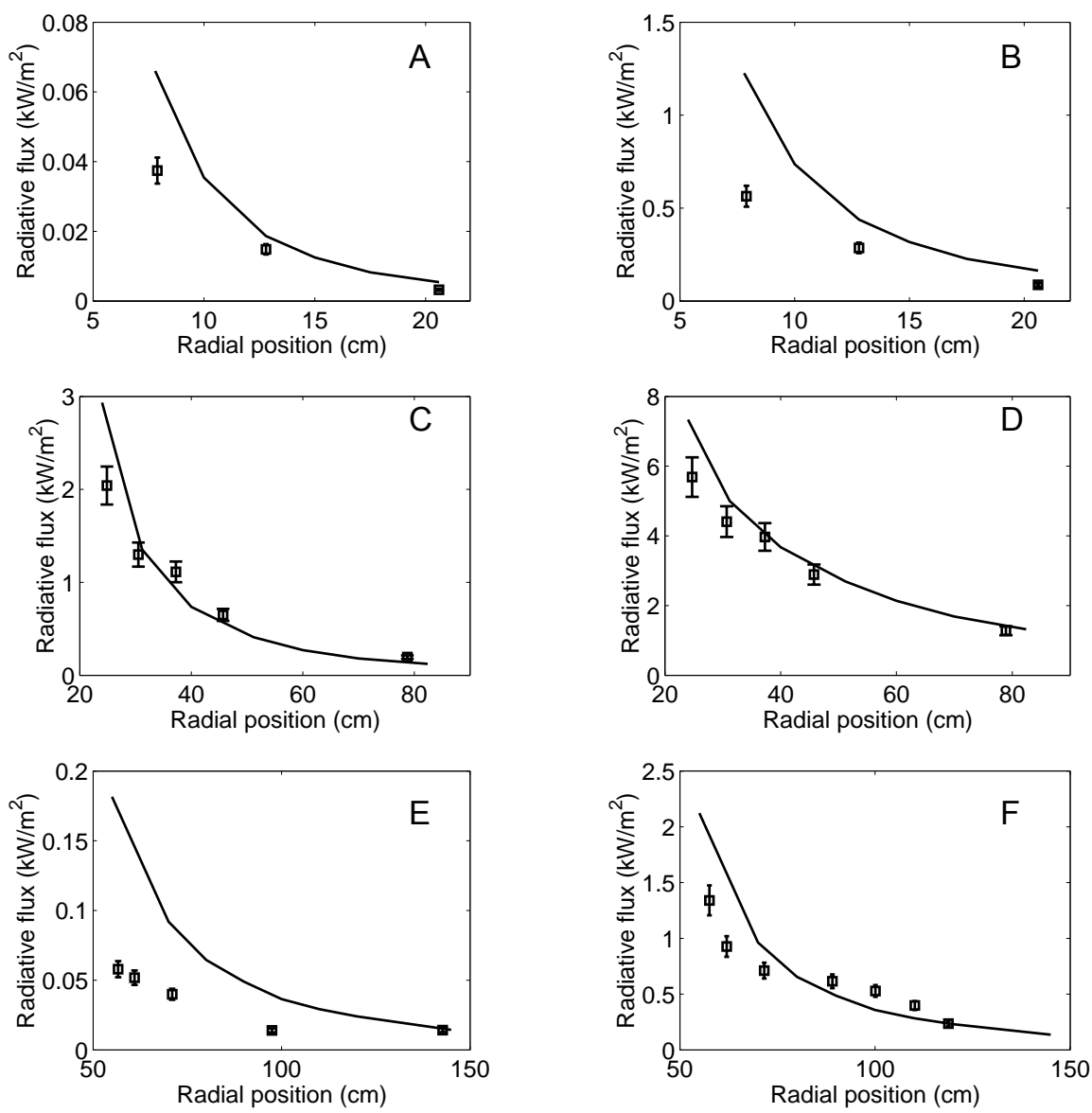

Fig. 2. - Comparison of the measured (squares) and predicted (lines) radial heat flux distributions outside the methane flames. 

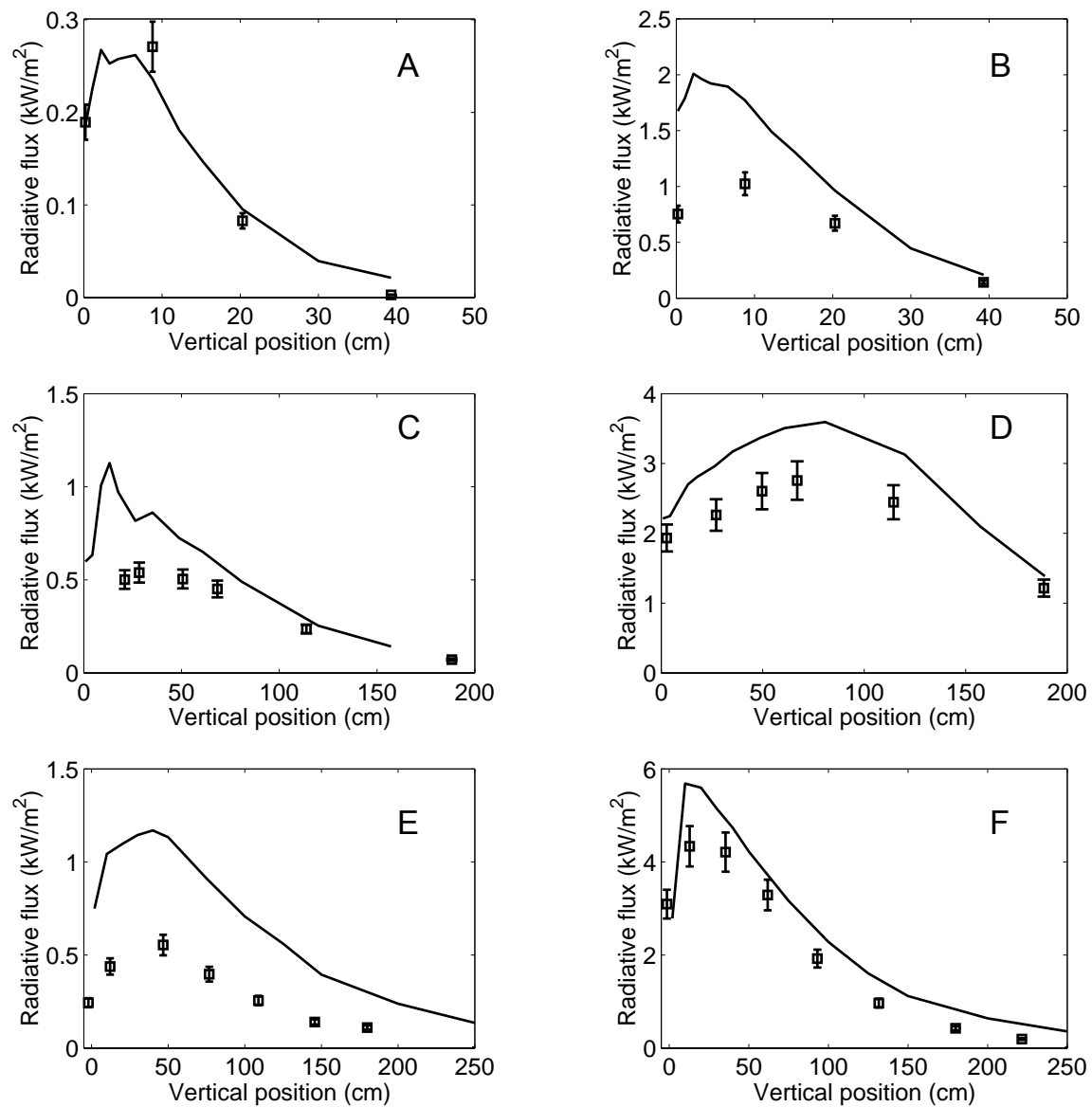

Fig. 3. - Comparison of the measured (squares) and predicted (lines) vertical heat flux distributions outside the methane flames.

Simulations of the methanol pool fires were carried out for four pool diameters: $1.0 \mathrm{~cm}$, $10 \mathrm{~cm}, 30 \mathrm{~cm}$ and $100 \mathrm{~cm}$. The computational grid was $50 \times 50 \times 100$ in all cases. 100 control angles and 6 bands were used for the radiation. A zero soot conversion factor was assumed. The burning rate of the pool was predicted using the pyrolysis model. Fig. 4 shows the instantaneous simulated flame shapes for the four cases. The amount of turbulence increases with diameter. The $1.0 \mathrm{~cm}$ flame is laminar and symmetric, but the $10 \mathrm{~cm}$ flame already contains asymmetric shapes due to the weak turbulence. The $30 \mathrm{~cm}$ and $100 \mathrm{~cm}$ flames have a substantial amount of fine structure. The measured burning rates and the corresponding predicted burning rates are given in Table 4. Table 4 also show the average values of the predicted radiative and convective heat fluxes on the pool surface. The convective heat transfer dominates at small diameters and radiation becomes more important at large diameters. The predicted burning rate of the smallest pool is lower than the experimental value and the other three predictions are $37 \%$ to $100 \%$ higher than measured. These errors are larger than the experimental uncertainty and, therefore, significant. The same conclusion can be done, if we compare with the regression rate data presented, for example in [14]. However, it should be recalled that 
no account of convection in the liquid pool has been taken, plus the convective heat transfer from the gas to the pool surface is not well resolved.

Fig. 5 shows a comparison of the measured (circles) and predicted (solid line) radial profiles of the mean temperatures above the $30 \mathrm{~cm}$ methanol pool. The effect of the burning rate on the temperatures was studied by repeating the simulation at a fixed burning rate, $14 \mathrm{~g} / \mathrm{m}^{2} / \mathrm{s}$. The corresponding calculated temperature profiles are shown with dashed lines. The comparisons are shown at three heights above the pool: $3.0 \mathrm{~cm}, 30$ $\mathrm{cm}$ and $60 \mathrm{~cm}$. Close to the pool surface, at a height of $3.0 \mathrm{~cm}$, the location of the predicted flame structure is too close to the pool edge. At the predicted burning rate, the temperatures near the center of the pool are much lower than the measured and fixed burning rate predictions. Higher above the pool surface, the calculated high burning rate is linked to the calculated temperatures, which are too high. It seems that the high temperatures in and above the flame zone are not the reason for the high burning rates, but a consequence. Possible sources of error are the lack of the liquid phase convection and the fact, that simple empirical correlations of convective heat transfer are being used to calculate the heat fluxes due to the flames touching down into the first grid cell above the liquid surface. Very high, moving spots of convective heat transfer were found to traverse the pool surface. This is also observed experimentally and remains a significant modeling challenge.

Table 4. - Summary of the methanol pool fire burning rates and predicted average heat fluxes to the pool surface.

\begin{tabular}{ccccc}
\hline \hline $\begin{array}{c}\text { Pool diameter } \\
(\mathrm{cm})\end{array}$ & $\begin{array}{c}\text { Measured burning rate } \\
\left(\mathrm{g} / \mathrm{m}^{2} / \mathrm{s}\right)\end{array}$ & $\begin{array}{c}\text { Predicted burning rate } \\
\left(\mathrm{g} / \mathrm{m}^{2} / \mathrm{s}\right)\end{array}$ & \multicolumn{2}{c}{ Predicted heat fluxes $\left(\mathrm{kW} / \mathrm{m}^{2}\right)$} \\
radiative & convective \\
\hline 1.0 & $59($ Corlett and $\mathrm{Fu}[15])$ & 32 & 5.7 & 175.5 \\
10 & 16 & 22 & 18.7 & 27.3 \\
30 & 13 & 28 & 35.4 & 25.9 \\
100 & 15 & 33 & 56.1 & 25.7 \\
\hline
\end{tabular}

The prediction of the liquid pool burning rate, starting from first principles, is an extremely challenging task. Individual numerical and physical errors are difficult to separate due to the strong coupling between the pool burning rate, gas phase heat release, gas temperatures and gas to solid heat transfer. Further validation should be carried out by trying to separate the different sources of errors. In addition, the entrainment rate of fresh air has a strong effect on the flame shape and temperatures. Comparison with experimentally measured velocities is therefore needed.

\section{CONCLUSIONS}

Large eddy simulations of turbulent diffusion flames have been carried out with several fire sizes and boundary conditions. Transport of thermal radiation was solved using the Finite Volume Method for radiation, combined with a simple wide band model for the spectral dependence of the absorption coefficient. The applications included the prediction of the radiative heat flux distributions outside the methane/natural gas flames, and the prediction of the methanol pool burning rates and flame temperatures. The model is capable of capturing the dynamical differences of flames ranging from the small burner to the scale close to the compartment fires. 
a)

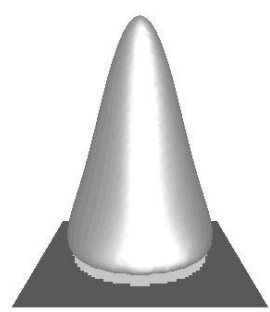

b)

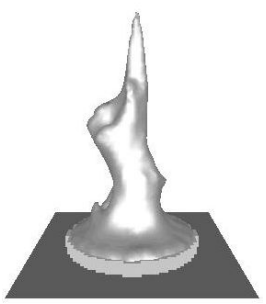

c)

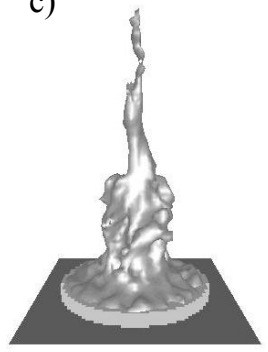

d)

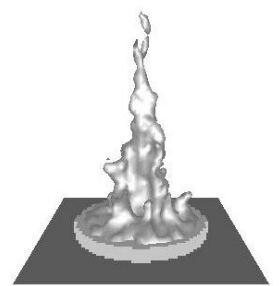

Fig. 4. - Instantaneous flame shapes of the simulated methanol pool fires. The diameters of the pools are a) $1.0 \mathrm{~cm}$, b) $10 \mathrm{~cm}, \mathrm{c}) 30 \mathrm{~cm}$ and d) $100 \mathrm{~cm}$.
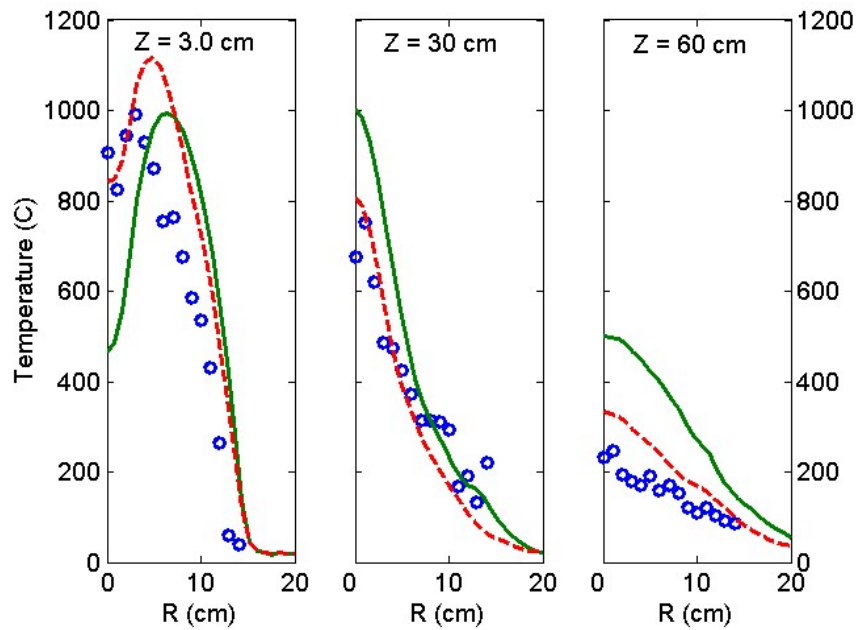

Fig. 5. - Comparison of the measured (circles) and predicted temperatures in the $30 \mathrm{~cm}$ methanol pool flame. The simulated temperatures are shown for both the predicted (solid lines) and the prescribed (dashed lines) burning rates.

Based on the shown applications we can conclude, that the current model is able to provide correct qualitative dependence between the pool size and burning rate, but more work is needed to improve the quantitative accuracy. The predicted radiative fractions of the methane/natural gas flames were shown to be systematically on the high side, probably due to the high temperatures close to the burner surface. However, the most accurate predictions of the heat flux distributions were found, where the heat fluxes were highest. This makes the model relevant for the fire safety engineering purposes. However, the physical resolution in the current applications was much better than what one can afford in the typical compartment fire simulations. The robustness of the current models must therefore be studied to ensure the predictive capability of the model in the compartment fires.

More detailed comparisons with measurements are needed to separate the individual sources of errors. The methods of calculating the band mean absorption coefficients should be studied more carefully. Also, the performance of the current combustion model is a subject of critical examination. 


\section{ACKNOWLEDGEMENTS}

The authors would like to thank Dr. Howard Baum of NIST for his guidance and Dr. Jason Floyd of NIST for his contribution to the model development.

\section{REFERENCES}

1. McGrattan, K.B., Baum, H.R., and Rehm, R.G. "Large Eddy Simulations of Smoke Movement," Fire Safety Journal, 30:161-178, 1998.

2. Rehm, R.G. and Baum, H.R. "The Equations of Motion for Thermally Driven, Buoyant Flows," Journal of Research of the NBS, 83:297-308, 1978.

3. McGrattan, K.B., Baum, H.R., Rehm, R.G., Hamins, A., Forney, G.P., Floyd, J.E. and Hostikka, S.. "Fire Dynamics Simulator (Version 2) - Technical Reference Guide," National Institute of Standards and Technology Report NISTIR 6783, 2001.

4. Huggett, C. "Estimation of the Rate of Heat Release by Means of Oxygen Consumption Measurements," Fire and Materials, 4:61-65, 1980.

5. Floyd, J.E., Baum, H.R., and McGrattan, K.B. "A Mixture Fraction Combustion Model for Fire Simulation Using CFD," Proceedings of the International Conference on Engineered Fire Protection Design, Society of Fire Protection Engineers, 2001, 279-290.

6. Siegel, R. and Howell, J.R. Thermal Radiation Heat Transfer, Hemisphere Publishing Corp., Philadelphia, $3^{\text {rd }}$ edition, 1992.

7. Grosshandler, W.L. "RADCAL: A Narrow-Band Model for Radiation Calculations in a Combustion Environment," National Institute of Standards and Technology. NIST Technical Note 1402, 1993.

8. Raithby, G.D. and Chui, E.H. "A Finite-Volume Method for Predicting Radiant Heat Transfer in Enclosures with Participating Media," Journal of Heat Transfer, 112(2):415-423, 1990.

9. Kim, S.H. and Huh, K.Y. "Assessment of The Finite-Volume Method and The Discrete Ordinate Method for Radiative Heat Transfer In a Three-Dimensional Rectangular Enclosure," Numerical Heat Transfer, Part B, 35:85-112, 1999.

10. International Organization of Standardization, Guide to the Expression of Uncertainty in Measurement, Geneve, Switzerland, 1993.

11. Hamins, A., Konishi, K., Borthwick, P. and Kashiwagi, T. "Global Properties of Gaseous Pool Fires", Proceedings of the Twenty-Sixth Sym. on Combust., 1996, 1429-1436.

12. Hamins, A., Klassen, M., Gore, J., and Kashiwagi, T. "Estimate of Flame Radiance via a Single Location Measurement in Liquid Pool Fires," Combustion and Flame, 86:223-228, 1991.

13. Pitts, W.M., Lawson, J.R., and Shields, J.R.. Report of Test FR 4014, "NIST/BFRL Calibration System for Heat-Flux Gages", August 6, 2001.

14. Drysdale, D., An Introduction to Fire Dynamics $\left(2^{\text {nd }}\right.$ ed), John Wiley and Sons, Chichester, 1999, p. 163.

15. Corlett, R.C. and Fu, T.M., "Some Recent Experiments with Pool Fires," Pyrodynamics, 4:253-269, 1966. 